\title{
Intralobar sequestration in the middle-aged and elderly adult: Recognition and radiographic evaluation
}

\author{
Gerard Petersen, MD, Ubaldo Martin, MD, Arun Singhal, MD, and Gerard J. Criner, MD, Philadelphia, Pa
}

I ntralobar sequestration is a rare cause of recurrent bronchitis, pneumonia, or hemoptysis that is often misdiagnosed both because of a lack of recognition of radiologic findings and a low expectation of the disease, particularly in middle-aged and elderly adults. Definitive therapy is surgical, and preoperative delineation of anatomy is essential. We present 2 cases from our institution that were atypical in presentation and used different radiographic imaging modalities. A review of the literature pertaining to presentations in older adults and radiographic delineation of these lesions follows.

\section{Clinical Summaries}

PATIENT 1. Patient 1 is a 79-year-old woman with a history of hypertension, bladder carcinoma treated by resection, and paroxysmal atrial fibrillation, with a 1-month history of episodic and progressive hemoptysis. She initially went to an outside institution with left-sided pleuritic chest pain, increasing sputum production, and 0.5 cup of hemoptysis in 24 hours. The patient denied fever, chills, weight loss, dyspnea, or trauma. Review of systems was significant for recurrent bronchitis. Her medications included metoprolol, hydrochlorothiazide, and digoxin. She was a nonsmoker and had no known occupational exposures.

Vital signs were notable for a temperature of $100.6^{\circ} \mathrm{F}\left(38.1^{\circ} \mathrm{C}\right)$. Physical examination revealed an elderly woman in no distress with rhonchi in the lower left lung field. Extremities were without clubbing or cyanosis. Results of laboratory investigation were significant for a white blood cell count of $18.3 \times 10^{3}$ cells $/ \mu \mathrm{L}$, a hemoglobin level of $10.0 \mathrm{~g} / \mathrm{dL}$, a platelet count of $442 \times 10^{3}$ cells $/ \mu \mathrm{L}$, and a prothrombin time of 12.4 seconds with an international normalized ratio of 1.0. Sputum analysis revealed moderate white blood cells and Staphylococcus aureus.

Fiberoptic bronchoscopy revealed bleeding from the left lower lobe, which prevented direct visualization of distal anatomy. No endobronchial lesions or masses were visualized. A chest radiograph revealed an infiltrate in the left lower lobe. Chest computed

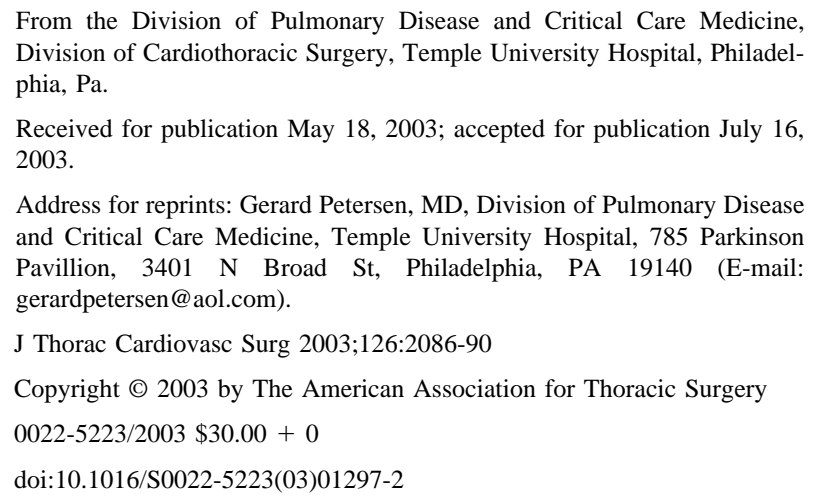

tomography (CT) revealed a contrast-enhancing mass in the left lower lobe (Figure 1). A chest CT and thoracic magnetic resonance angiography (MRA) were interpreted as showing a saccular thoracic aneurysm with dissection into the lung parenchyma.

The patient was transferred to our institution for possible surgical intervention. Our review of the MRA showed a contrastenhancing mass in the left lower lobe with a feeding vessel off the descending thoracic aorta (Figure 2). Retrograde aortography revealed a vessel off the descending thoracic aorta feeding into a dilated space with extravasation (Figure 3).

The patient underwent surgical resection. During the operation, the left lower lobe was found to be adhered to the descending thoracic aorta. No surgical plane could be developed. The left lower lobe and a $6-\mathrm{cm}$ portion of the descending thoracic aorta were resected en bloc, and the aorta was replaced with a homograft. The gross specimen revealed a 1-mm feeding vessel that originated from a $3-\mathrm{cm}$ defect in the descending thoracic aorta and fed into a cystic mass containing pus and old thrombus surrounded by cystic lung parenchyma. Histopathologic analysis revealed chronic adhesive pleuritis, a zone of chronic abscess and hematoma that merged gradually with the surrounding inflamed lung parenchyma, and remnant airways with cystic dilatation. The feeding vessel off the descending thoracic aorta was confirmed to be arterial by an elastic tissue stain. The final pathologic diagnosis was intralobar sequestration of the left lower lobe. According to our review of the literature, this patient is the oldest to be reported to have intralobar sequestration.

PATIENT 2. Patient 2 is a 41 -year-old woman without significant medical history who came to an outside institution with a 1-month history of cough, fever, and fatigue. An admission chest radiograph revealed a right lower lobe density, and a subsequent chest CT revealed a lobulated right lower lobe mass. A fine needle aspirate was performed; this revealed benign alveolar tissue with fibrosis, dense lymphoplasmacytic inflammation, and occasional multinucleated giant cells. She was referred to our institution for a second opinion. Her review of systems revealed no recurrent bronchitis or pneumonia.

Physical examination revealed bilateral clear lung fields and otherwise normal results. Laboratory investigation revealed a white blood cell count of $6.2 \times 10^{3}$ cells $/ \mu \mathrm{L}$, a hemoglobin level of $13.6 \mathrm{~g} / \mathrm{dL}$, and a platelet count of $305 \times 10^{3}$ cells $/ \mu \mathrm{L}$.

A chest radiograph revealed a solid density in the posterior right lower lobe. Chest CT revealed a lobulated 4-cm mass and consolidated lung tissue along the posterior lateral aspects of the right hemithorax without evidence of contrast enhancement.

This patient underwent magnetic resonance imaging (MRI) and MRA with and without gadolinium. Scans revealed a nonenhancing isointense mass measuring $5.0 \times 4.6 \times 6.0 \mathrm{~cm}$ in the right cardiophrenic angle and containing an air-fluid level with an arterial feeding vessel from the infradiaphragmatic aorta. The 


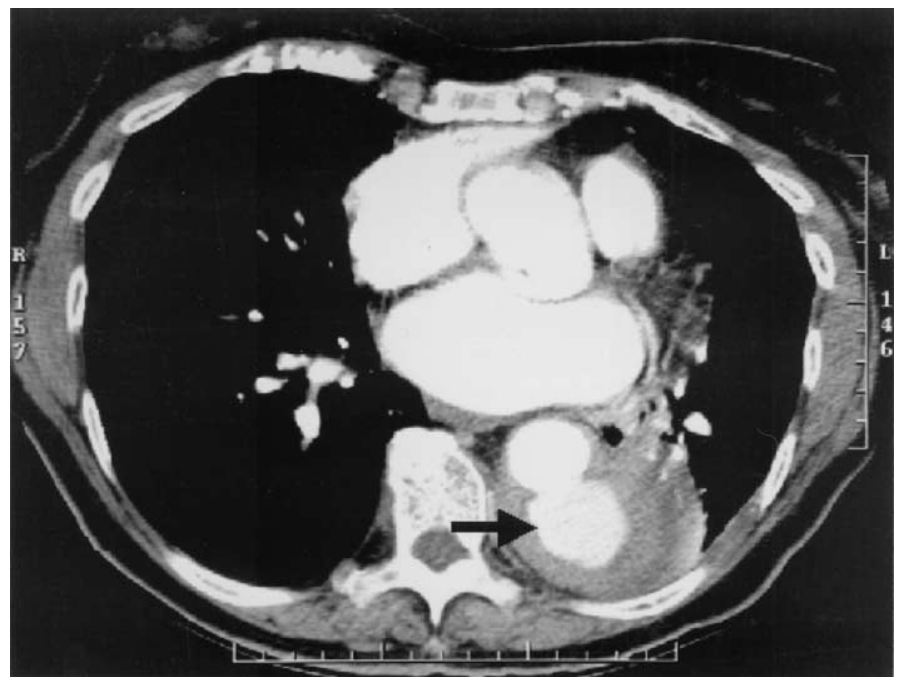

Figure 1. Contrast chest CT with contrast-enhancing mass in left lower lobe (arrow).

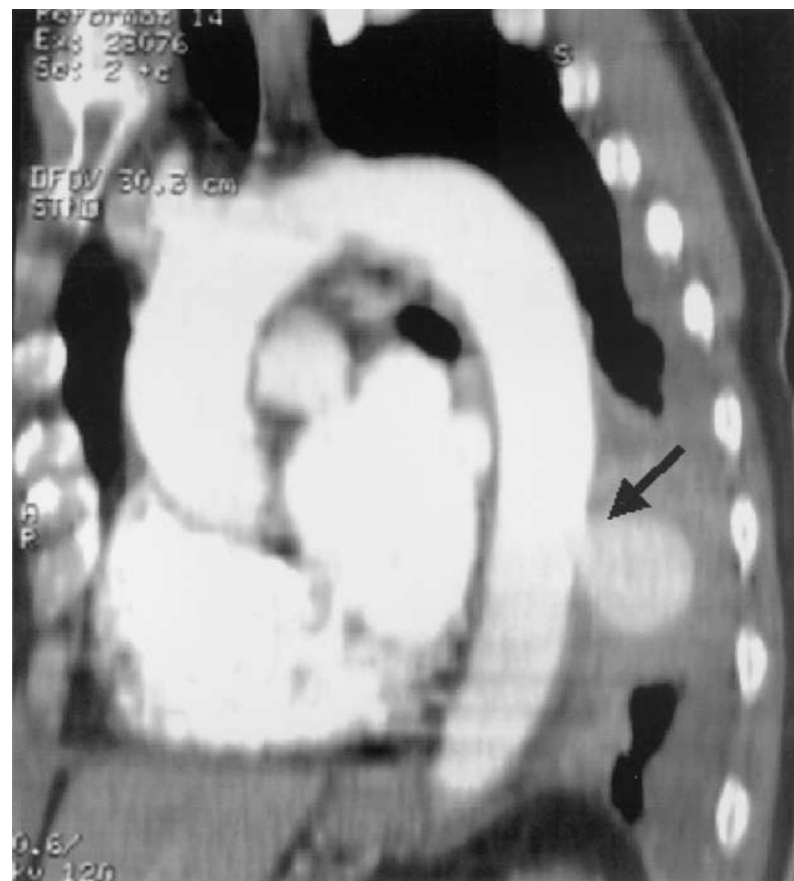

Figure 2. MRA of thorax with contrast-enhancing mass in left lower lobe with feeding vessel (arrow) off descending thoracic aorta.

venous drainage was through the right inferior pulmonary vein (Figure 4).

The patient underwent a right thoracotomy with resection of the intralobar sequestration and ligation of the feeding vessel that traversed the diaphragm. The gross specimen revealed a thickwalled blood vessel feeding into a segment of lung. On crosssection, there were yellow, necrotic confluent masses with cystic

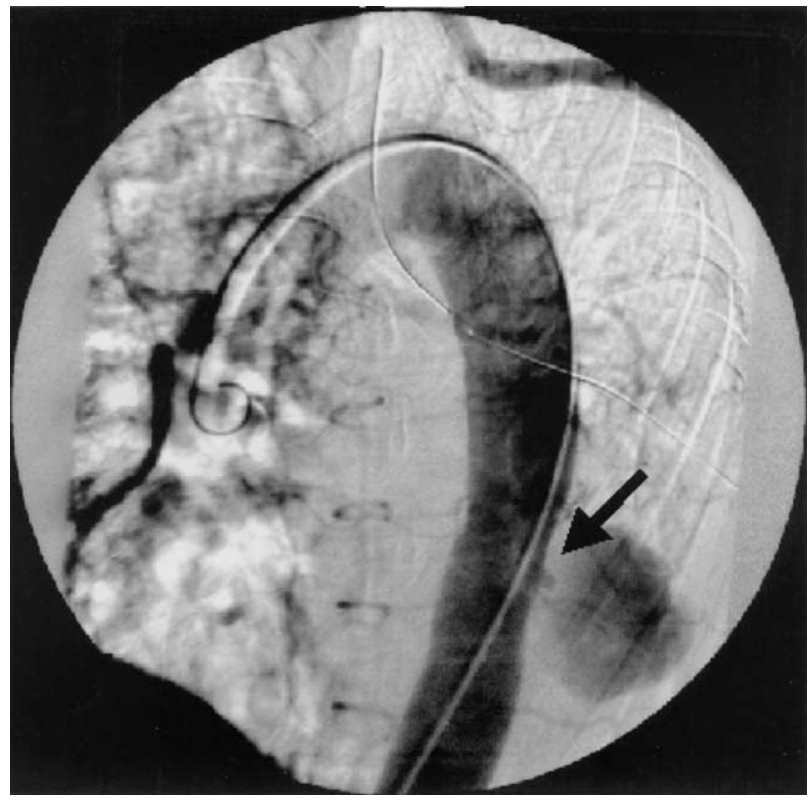

Figure 3. Retrograde aortography with vessel (arrow) off descending thoracic aorta feeding into dilated space with extravasation.

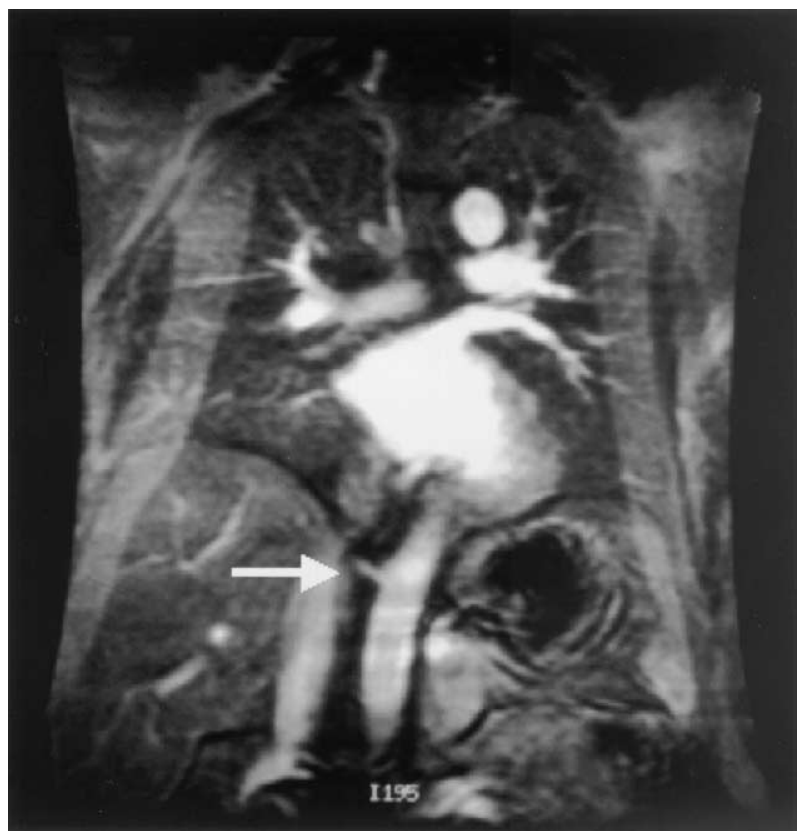

Figure 4. MRA of thorax revealing large feeding vessel (arrow) from subdiaphragmatic descending aorta.

spaces. The histopathologic examination showed dilated alveolar spaces with prominent type II pneumocytes, abundant foamy macrophages and lymphocytic aggregates filling the alveolar and interstitial spaces, and areas of focal hemorrhage and pleural fibrosis. The final pathologic diagnosis was intralobar sequestration of the right lower lobe. 
TABLE 1. Reports in the literature of patients older than $\mathbf{4 0}$ years with intralobar sequestration

\begin{tabular}{|c|c|c|c|}
\hline Reference & Age (y) & Sex & Clinical findings \\
\hline Croyle and Estrera ${ }^{5}$ & 49 & Male & Asymptomatic, retrocardiac mass \\
\hline Choplin and Siege ${ }^{6}$ & 57 & Male & Fever, cough \\
\hline \multirow[t]{5}{*}{ Ikezoe et $\mathrm{al}^{7}$} & 58 & Male & Cough, sputum, hemoptysis \\
\hline & 71 & Male & Asymptomatic \\
\hline & 56 & Male & Asymptomatic \\
\hline & 48 & Male & Asymptomatic \\
\hline & 42 & Male & Shortness of breath \\
\hline Lee and Nemcek ${ }^{8}$ & 43 & Male & Symptomatic, masses on chest radiograph \\
\hline Lewis and Tsour ${ }^{9}$ & 66 & Male & Dyspnea \\
\hline \multirow[t]{2}{*}{ Petersen et al (current report) } & 41 & Female & Fever, cough \\
\hline & 79 & Female & Hemoptysis \\
\hline Singh and Nath 10 & 53 & Male & Hemoptysis \\
\hline Tsunezuka and Sato 11 & 75 & Female & Asymptomatic, abnormal shadow \\
\hline \multirow[t]{2}{*}{ Yamanaka et al ${ }^{12}$} & 46 & Male & Cough, sputum, hemoptysis \\
\hline & 68 & Female & Dyspnea, hemoptysis \\
\hline
\end{tabular}

\section{Discussion}

The term sequestration, first used in 1946 by Pryce, ${ }^{1}$ refers to an abnormality in which a segment of bronchopulmonary tissue has an anomalous systemic arterial blood supply. Two types have been described, intralobar and extralobar. Intralobar sequestration, which accounts for $75 \%$ of cases, is a segment of lung contained within the normal visceral pleura. In contrast, in extralobar sequestration the bronchopulmonary tissue is outside the visceral pleura and may be enveloped in its own pleura.

Intralobar sequestration is typically diagnosed in pediatric or adolescent patients, with equal gender prevalence, but is also diagnosed in adults. Presentation varies from the incidental finding of a mass in a symptom-free patient to symptoms of recurrent bronchitis or pneumonia. Patients may also have hemoptysis, as did patient 1 . In contrast to extralobar sequestration, intralobar sequestration is infrequently associated with other congenital abnormalities. It is theorized that the pathogenesis of sequestration is the result of an accessory lung bud that develops from the ventral primitive foregut as it migrates caudally. The sequestration retains the splanchnic blood supply, subsequently receiving systemic arterial supply instead of the normal pulmonary arterial supply.

Intralobar sequestration is diagnosed at age 20 years or younger in approximately $50 \%$ to $60 \%$ of cases, and it is rarely found in patients older than 40 years. ${ }^{2,3}$ Savic and colleagues ${ }^{4}$ noted that a small number of patients (15\%) do not have symptoms when the lesion is discovered. Our review of the literature, including our 2 cases, yielded 15 reported cases of intralobar sequestration in patients older than 40 years. ${ }^{6-12}$ The different presentations are listed in Table 1. The number of symptom-free patients, 6 of 15 (40\%), is higher than reported by Savic and colleagues. ${ }^{4}$ Perhaps this reflects a higher likelihood for older adults to obtain chest radiographs for unrelated reasons. We also noted a trend toward male predominance among older adults, although intralobar sequestration is reported to have equal gender prevalence. From our review of the literature, our patient is the oldest reported to have intralobar sequestration. Savic and colleagues ${ }^{4}$ reported the case of an 81-year-old woman with extralobar sequestration.

In older adults the differential diagnosis includes entities similar to those in pediatric and adolescent populations: pneumonia, lung abscess, bronchiectasis, empyema, bronchogenic cysts, and pulmonary or mediastinal neoplasm. However, concerns for pulmonary neoplasms weigh heavier with older adults. In addition, thoracic aortic aneurysms enter the differential diagnosis, as illustrated patient 1 and as reported by Croyle and Estrera. ${ }^{5}$ Some authors have proposed that presentations in older adults support the theory that intralobar sequestration represents an acquired lesion related to bronchial obstruction, pneumonia, pulmonary artery occlusion, pleuritis, pulmonary ligament thickening, and parasitization of pulmonary ligament arteries. ${ }^{13}$

The typical location is in the medial or posterior basal segments of the left lower lobe. It has been reported that $95 \%$ of cases are located in the lower lobes, with the remainder in the mid and upper lobes. ${ }^{13}$ Although typically there is no communication between the tracheobronchial tree and sequestered lung, cases have been described in which there is a connection with collateral ventilation aerating the sequestered tissue. Infection and inflammation in the sequestered portion of lung may lead to tissue breakdown and subsequent communication with the normal tracheobronchial tree. However, cases have been described in which there is no histopathologic evidence of inflammation, suggesting that the communication may be part of the original abnormality. ${ }^{14}$

Pathologic specimens usually reveal distinct demarcation from the surrounding lung parenchyma. The gross specimen consists of cystic spaces admixed with denser fibrotic lung tissue. The cystic spaces may be filled with pus, mucus, or blood. Histopathologic appearance may reveal a fibrous capsule, cysts consisting of dilated bronchi, denuded respiratory epithelium, alveolar spaces with intraluminal macrophages, and surrounding interstitial fibrosis with acute and chronic inflammatory infiltrate.

The arterial supply derives from the aorta, predominantly the descending thoracic aorta (75\%) and less frequently the abdominal aorta. Other derivations have been reported. One study of 105 cases revealed that 74 were derived from the descending thoracic aorta, 25 from the abdominal aorta, 5 from the intercostal arteries, and 1 from the aortic arch. ${ }^{2}$ Venous outflow is predominantly through the pulmonary venous system $(96 \%)$.

Therapy consists of surgical resection. Preoperative delineation of the anomalous vasculature minimizes fatal outcomes from hem- 
orrhage caused by accidental division of the systemic artery. The traditional approach has been evaluation with retrograde aortography. However, a noninvasive approach is more desirable if it provides comparable information. Techniques used for imaging bronchopulmonary sequestration have been ultrasonography, scintigraphy, CT, and MRA.

CT. CT adequately displays lung parenchyma and can demonstrate the anomalous systemic artery in about $80 \%$ of cases. $^{7}$ Ikezoe and associates ${ }^{7}$ investigated 24 cases from several institutions of pulmonary sequestration, both intralobar and extralobar, which were confirmed either intraoperatively or by pathologic specimen. CT revealed the systemic artery in 16 of 24 cases overall and in 13 of 16 cases of intrapulmonary sequestration specifically. In 2 patients in whom only one systemic artery was identified on CT, two or more anomalous systemic arteries were demonstrated via aortography or surgery. Most scans were obtained with $10-\mathrm{mm}$ collimation at $10-\mathrm{mm}$ intervals with intravenous contrast.

Ko and colleagues ${ }^{15}$ noted that helical CT could facilitate the display of the aberrant artery relative to conventional CT. This is achieved by volume acquisition through slice reconstruction with narrow intervals and multiplanar and 3-dimensional reformatting. Venous drainage may not be as clearly shown on CT. ${ }^{7}$ However, an obviously enlarged azygous system (azygous plus hemiazygous diameters $>10$ $\mathrm{mm}$ ) associated with a posterobasal chest lesion may suggest the diagnosis of bronchopulmonary sequestration.

MRI and MRA. The use of MRI to define the systemic arterial supply was first reported by Oliphant and cowork$\mathrm{ers}^{16}$ in 1987 . Other authors have since reported the utility of MRI. Breath-hold contrast-enhanced MRA can offer an excellent display of the aberrant artery without flow or respiratory artifact. ${ }^{17} \mathrm{~A}$ reported limitation of MRI is that small anomalous vessels may not be detected. ${ }^{3}$ Magnetic resonance cannot delineate focal thin-walled cysts or the emphysematous changes of sequestration as well as CT. However, contrast-enhanced MRA has been shown to be useful in evaluating the thoracic aorta and pulmonary vasculature. ${ }^{17,18}$ Multiphase scanning after gadolinium administration allows demonstration of arteries and veins separately and hence improved visualization of the venous drainage in pulmonary sequestration. Two-dimensional MRA is limited by low spatial resolution and turbulent flow.

Zhang and colleagues ${ }^{19}$ recently reported on 3 patients with suspected pulmonary sequestration who were examined with contrast enhanced 3-dimensional MRA and MRI images. The radiographic images were compared with surgical findings and were consistent in all 3 cases. The authors noted that previous reports had used 2-dimensional MRA, which has several limitations: (1) the necessity of breath holding to maximize flow-related enhancement and to eliminate respiratory artifact, (2) the need for correct orientation of the plane of acquisition, and (3) flow-dependent signal that may fail to detect small vessels with low flow or inadequately describe large vessels with turbulent flow. Contrast-enhanced 3-dimensional MRA does not require proper orientation of the plane of acquisition. Additionally, imaging is not flow-dependent, thus allowing improved visualization of smaller vessels.

Retrograde aortography. Retrograde aortography has been the traditional approach to diagnosing pulmonary sequestration by defining an aberrant systemic artery. According to one report, multiple arteries supplied the lesion in $16 \%$ of cases, ${ }^{13}$ and a single aortic branch may divide into several tributaries before entering the sequestration. ${ }^{4,20}$ This information has been argued to favor the use of retrograde aortography relative to other imaging modalities. However, retrograde aortography remains a 2-dimensional, time-offlight imaging modality that may also share the limitations of plane of acquisition.

Conclusions. Because of the relatively small prevalence of this disease entity, a controlled study comparing the different diagnostic imaging modalities is extremely unlikely. The most important tool remains a heightened awareness of this disease process in older adults. Older patients appear to have symptoms similar to those in younger populations with a trend toward more frequent asymptomatic presentations detected on radiographs. A review of the literature and our 2 cases suggests that MRA, in particular contrast-enhanced 3-dimensional MRA, can play an important role in the diagnosis and preoperative evaluation of patients with bronchopulmonary sequestration, perhaps obviating the need for retrograde aortography in specific cases.

\section{References}

1. Pryce DM. Lower accessory pulmonary artery with intralobar sequestration of lung: a report of seven cases. J Pathol Bacteriol. 1946;58: 457-67.

2. Turk LN III, Lindskog GE. The importance of angiographic diagnosis in intralobar pulmonary sequestration. J Thorac Cardiovasc Surg. 1961;41:299-305.

3. Gustafson RA, Murray GF, Warden HE, Hill RC, Rozar GE. Intralobar sequestration: a missed diagnosis. Ann Thorac Surg. 1989;47:841-7.

4. Savic B, Birtel FJ, Tholen W, Funke HD, Knoche R. Lung sequestration: report of seven cases and review of 540 published cases. Thorax. 1979;34:96-101

5. Croyle P, Estrera AS. Bronchogenic cysts and intralobar sequestration mimicking thoracic aortic aneurysm. South Med J. 1982;75:1267-8.

6. Choplin RH, Siegel MJ. Pulmonary sequestration: six unusual presentations. AJR Am J Roentgenol. 1980;134:695-700.

7. Ikezoe T, Murayama S, Godwin JD, Done S, Verschakelen J. Bronchopulmonary sequestration: CT assessment. Radiology. 1990;176:375-9.

8. Lee RA, Nemcek AA. Complex mass at right lung base. Chest. 1998;94:397-9.

9. Lewis MM, Tsour E. A 66-year-old man with dyspnea, left lower lobe infiltrate, and abnormal imaging. Chest. 2000;117:1782-6.

10. Singh S, Nath H. A 53-year-old man with hemoptysis. Chest. 2001; 1120:298-301.

11. Tsunezuka Y, Sato H. Intralobar pulmonary sequestration with three aberrant arteries in a 75-year-old patient. Chest. 1998;114:936-8.

12. Yamanaka A, Hirai T, Fujimoto T, Hase M, Noguchi M, Konishi F. Anomalous systemic arterial supply to normal basal segments of the left lower lobe. Ann Thorac Surg. 1999;68:332-8. 
13. Stocker T, Malczak H. A study of pulmonary ligament arteries: relationship to intralobar pulmonary sequestration. Chest. 1984;86:611-5.

14. Iwai K, Shindo G, Hajikano H. Intralobar pulmonary sequestration, with special reference to developmental pathology. Am Rev Respir Dis. 1973;107:911-20.

15. Ko SF, Ng SH, Lee TY, Wan YL, Liang CD, Lin JW, et al. Noninvasive imaging of bronchopulmonary sequestration. AJR Am J Roentgenol. 2000;175:1005-12.

16. Oliphant L, McFadden RG, Carr TJ, Mackenzie DA. Magnetic resonance imaging to diagnose intralobar pulmonary sequestration. Chest. 1987;91:500-2.
17. Au VW, Chan JK, Chan FL. Pulmonary sequestration diagnosed by contrast enhanced three-dimensional MR angiography. Br J Radiol. 1999;72:709-11.

18. Watanabe Y, Dohke M, Okumura A. Dynamic subtraction contrast enhanced MR angiography: technique, clinical applications, and pitfalls. Radiographics. 2000;20:135-53.

19. Zhang M, Zhu J, Wang Q, Shang D. Contrast enhanced MR angiography in pulmonary sequestration. Chin Med $J$ (Engl). 2001;114: 1326-8.

20. Kent M. Intralobar pulmonary sequestration. Prog Pediatr Surg. 1991; 27:84-91.

\title{
Staged dilation and stenting for long segmental tracheobronchial stenosis caused by tuberculosis
}

\author{
Pei-Ming Huang, MD, ${ }^{a}$ Jin-Shing Chen, MD, ${ }^{a}$ Hsao-Hsun Hsu, MD, ${ }^{a}$ Yih-Leong Chang, MD, ${ }^{b}$ Chung-Wei Chen, MD, \\ Nai-Chuan Chien, MD, and Yung-Chie Lee, MD, PhD, ${ }^{a}$ Taipei, Taiwan
}

$\int$ ndobronchial tuberculosis is defined as tuberculosis of the tracheobronchial tree (TTBT) with microbiological evidence. The estimated prevalence of this complication in patients with pulmonary tuberculosis is $10 \%$ to $40 \%$, and more than $90 \%$ of patients with endobronchial tuberculosis have some degree of bronchial stenosis. ${ }^{1}$ TTBT, in general, is known for its propensity for longer segmental involvement. Despite adequate antituberculosis therapy, tracheobronchial stenosis may develop. ${ }^{2}$ Although several therapeutic approaches, including antituberculous chemotherapy combined with steroids, balloon dilation, laser photoresection, and surgical resection, have been attempted in these patients, the results are largely unsatisfactory. ${ }^{1,2}$ Long-segment tracheobronchial stenosis (LTBS) provides a complex challenge to stenting, ${ }^{3}$ but staged stenting can offer an attractive alternative to standard LTBS therapy.

We report a method of staged stenting used to periodically dilate the stenotic area, and this method is contrary to accepted recommendations for the insertion of single stent or double stents. Because the site is problematic to manage and reobstruction of the airway is likely, repeated dilation and stenting is an appealing option.

\footnotetext{
From the Departments of Surgery and Pathology, ${ }^{\mathrm{b}}$ Division of Thoracic Surgery, National Taiwan University Hospital, Taipei, Taiwan.

Received for publication May 14, 2003; accepted for publication July 30, 2003.

Address for reprints: Yung-Chie Lee, MD, PhD, Division of Thoracic Surgery, Department of Surgery, National Taiwan University Hospital, No. 7, Chung-Shan S. Rd, Taipei, Taiwan (E-mail: wuj@ha.mc.ntu.edu.tw).

J Thorac Cardiovasc Surg 2003;126:2090-2

Copyright $\odot 2003$ by The American Association for Thoracic Surgery $0022-5223 / 2003 \$ 30.00+0$

doi:10.1016/j.jtcvs.2003.07.001
}

\section{Clinical Summary}

From February 2001 through May 2002, 3 patients (ages 28, 44, and 62 years; 2 women and 1 man) with tuberculosis had severe airway obstruction and were treated at our institution. The diagnosis of LTBS was considered if chest radiograph or sputum culture was positive for tuberculosis and bronchoscopy and computed tomography (CT) revealed stenosis of the airway. Following failure of 6 months of medical therapy to relieve stenosis, surgical intervention was performed to relieve the compromised airway after the sputum cultures became negative for tuberculous bacilli. A midline tracheostomy was performed first. After 1 to 2 weeks all patients fasted for 8 hours before airway stenting preparation. The Bakes common bile duct dilators (Pro-Med, Instrumente $\mathrm{GmbH}$, Tuttlingen, Germany) were employed for progressive dilation under a flexible guided bronchoscope through the tracheostoma; anesthesia was continued through the oral endotracheal tube simultaneously (Figure 1). The stenotic lengths were as follows: (1) $1 \mathrm{~cm}$ in the trachea portion and $2 \mathrm{~cm}$ in the left main bronchus; (2) $2 \mathrm{~cm}$ in the trachea portion and $3 \mathrm{~cm}$ in the left main bronchus; and (3) $4 \mathrm{~cm}$ in the left bronchus by bronchoscopic examination during the operation. A measured length of silicone endotracheal tube or chest tube was used to bypass the stenotic area as the connecting conduits to the stoma portion of conventional tracheostomy tube, which we labeled the "tracheostomy stent" (Figure 2).

Because the tracheostomy stent was easily removed and repeatedly inserted, the patients could receive repeated dilation by employing a temporary tracheostomy stent via tracheostoma and flexible bronchoscopy until the stenotic lumen become larger. Fiberoptic bronchoscopy was performed every month until there was no subsequent change in the endobronchial lesions, and then the dynamic Y stents (Willy Rusch AG, Kernen, Germany) were implanted in the individual patients 1,2 , and 8 months later, respectively. During this period, the respective numbers of repeated dilations were 2,1 , and 3 .

In recent follow-up study, the first patient had minimal whitish sputum, and no granulation tissue was found in the last bronchos- 